\section{$5,7-61$ \\ 3,9678}

$N 91-17576$

\title{
Formally specifying the logic of an automatic guidance controller
}

\author{
David Guaspari
}

Odyssey Research Associates 


\section{Truth arises more readily from error than from confusion.}

Francis Bacon

Novum Organum 
The Penelope project:

- Interactive, incremental, tool for formal verification of Ada programs (Larch/Ada specifications).

- Structure or ordinary text editor

- Permits development of program and proof in concert, "reuse by replay"

- Covers large subset of sequential Ada.

- Mathematically based. 
Problem: specify "logic" of experimental Automatic Guidance Control System for a 737

- Pilot requests kind and degrees of automatic assistance

- Requests may be honored, disallowed, "put on hold

- Responses must be displayed 
Work-in-progress: Larch/Ada specification

- Formal specification of Ada code

- Goals: precise; intelligible to designers and implementors

- Currently wrong, but clear

Related work

- Original code (CSC)

- Experiment in redesign (NASA) 


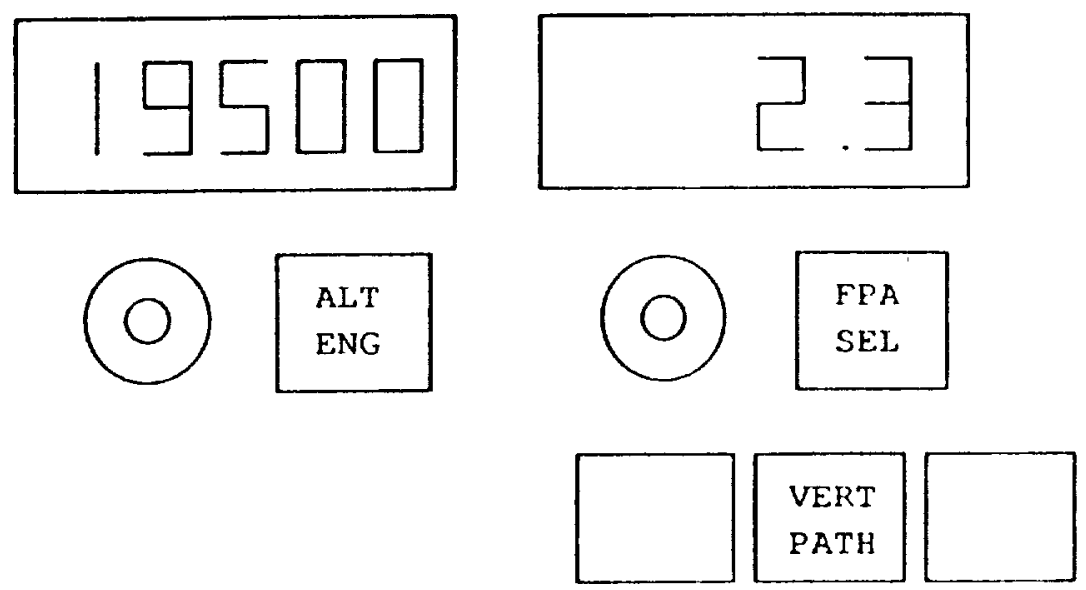




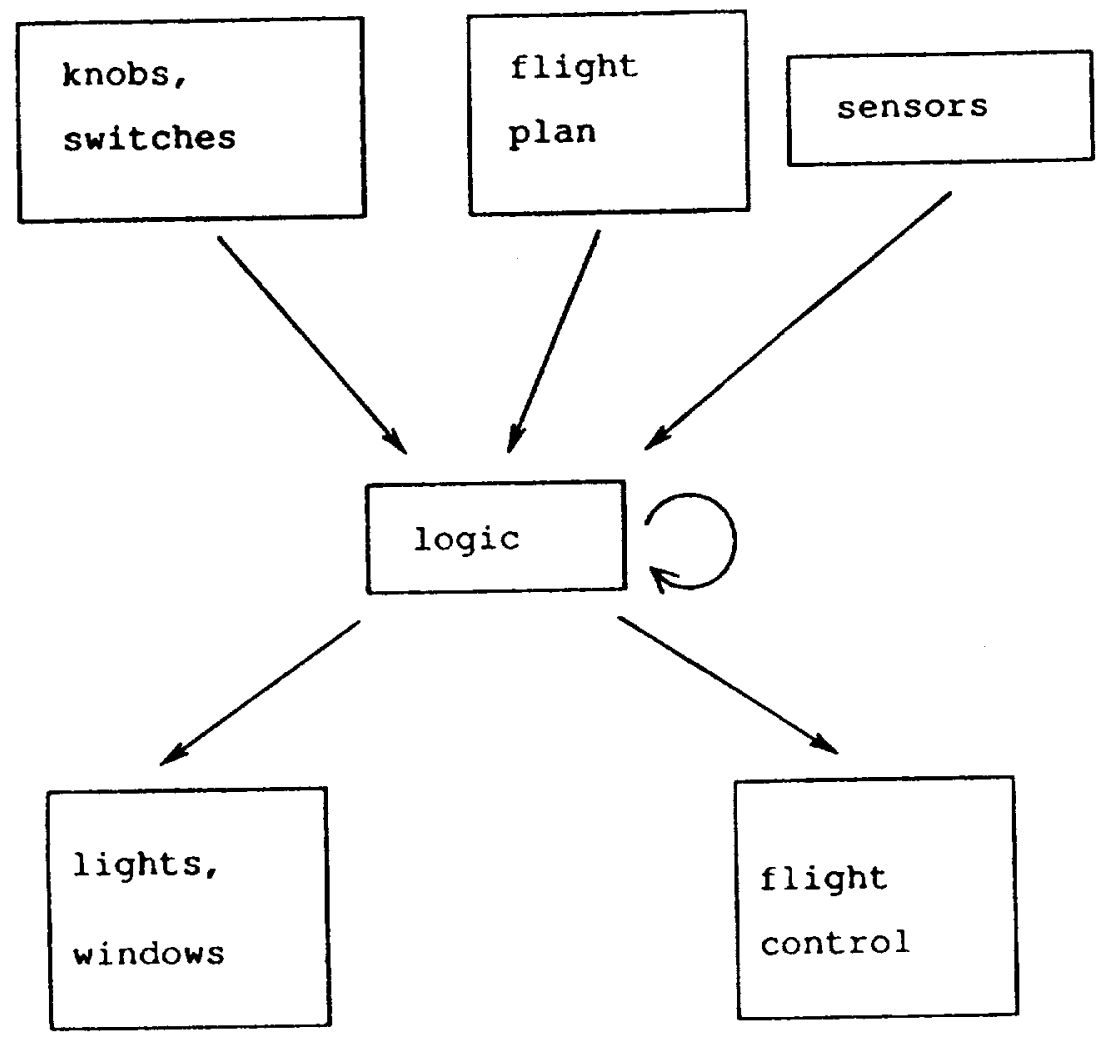


Some failures of informal description

1. Ambiguous: "Select" a switch vs. "select" a mode.

2. Incomplete: "CAS ENG may be engaged independent of all other AGCS modes except TIME PATH."

3. Contradictory:

- FPA ... cannot be deselected directly.

- [if] ... appropriate selection of the FPA SEL ... switch returns the mode to the off state... 


\section{Larch/Ada specifications: "two-tiered"}

- Mathematical part (Larch Shared Language): defines vocabulary

\section{- Interface part (Larch/Ada): uses vocabu- lary to specify code}


Example: specifying executable addition

Mathematical part: defines mathematical + on Int, the (infinite) domain of mathematical integers

Interface part: Specifying evaluation of $\mathrm{x}+\mathrm{y}$

- Type integer is "based on" Int.

- Return value $(x+y)$ if

$$
\min \leq(\mathrm{x}+\mathrm{y}) \leq \max .
$$

No side effects.

- Otherwise, raise numeric_error. No side effects. 


\section{The mathematical part}

States: AGCS_state, Sensor_state, etc.

Actions:

$$
\begin{gathered}
\{\text { alt_eng_switch, } \ldots \text {, alt_eng_knob(i),... } \\
\text { alt_capture, } . .\}
\end{gathered}
$$

Modes:

$$
\{\text { alt_eng,fpa_sel,vert_path,... }
$$

Transition operation:

AGCS_state, Action, ... $\rightarrow$ AGCS_state

Observers: active2d, display, ... 


\section{Building mathematical part (the AGCS states)}

AgcsStructure : trait

AGCS_state record of

(on: Bool,

modes: Set_of_modes,

engaged: Engagement_status,

setting: Value_settings,

window: Window_array)

includes Set(Mode,Set_of_modes)

\section{introduces}

transition:

AGCS_state, Action, Sensor_state,

Flight_plan $\rightarrow$ AGCS_state

initial_on_state: $\rightarrow$ AGCS_state

asserts 
Description of mode changes caused by switches:

- Is the mode directly deselectable?

- What mode changes result?

- Under what conditions is the mode directly selectable?

- What mode changes result? 
Building mathematical part (mode changes)

HorPathSwitch : trait

includes SwitchShell\{hor_path\}

asserts for all

[agcsmodes: Set_of_modes,

pl: Flight_plan,

sens: Sensor_state]

hor_path_deselectable

hor_path_selectable(agcsmodes,pl) =

(auto $\in$ agcsmodes) $\wedge$ active $2 \mathrm{~d}(\mathrm{pl})$

hor_path_selection_result(agcsmodes,sens,pl) =

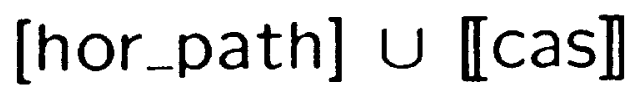

hor_path_deselection_result(agcsmodes) =

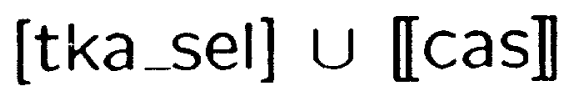


Intuitive description of window status (chosen vs. current):

- The $w_{-}$knob makes the corresponding $w$ window chosen.

- Any action selecting the $w$ mode makes the $w$-window chosen.

- Any action deselecting the $w$ mode makes the $w$-window current.

- Any other action leaves the status of the $w$-window unchanged. 
Building the mathematical part (window changes)

\section{StatusShell : trait}

imports AgcsStructure

introduces

\#.component :

Window_array $\rightarrow$ Window_status

md: $\rightarrow$ Mode

knob : Value $\rightarrow$ Action

asserts for all [agcs:AGCS_state, ...]

\section{abbreviation}

agcs' $==$ transition(agcs,act,sensor,plan)

agcs'. window.component $=$

if $m d \in$ agcs'.modes - acgs.modes

then chosen

elsif md $\in$ agcs.mode - agcs'.modes

then current

elsif act $=k n o b(i)$ then chosen

else agcs.window.component

Example: StatusShell\{alt,alt_eng,Airspeed 
Design of the code:

- Packages panel_logic, display_manager, sensor_data, flight_plan, flight_control.

- State of panel_logic based on AGCS_state, etc.

- Actions $\mapsto$ procedures of panel_logic:

- read state of panel_logic, sensor_data, flight_plan

- modify states of panel_logic, display_manager, flight_control

- Consistent with polling, interrupts, etc. 


\section{Specifying the code:}

--I WITH TRAIT AgcsLogic, AgcsProperties,

$--1 \quad$ LogicalDisplay

--1 WITH sensor_data, flight_plan,

-1 display_manager, flight_control

with sensor_data_types; use sensor_data_types; package panel_logic

--1 BASED ON AGCS_state

-1 INVARIANT

--1 panel_logic.on $\rightarrow \operatorname{good}($ panel_logic)

-1 INITIALLY not panel_logic.on

end panel_logic; 
procedure att_cws_switch;

--1 WHERE

--1 GLOBALS IN panel_logic

- I GLOBALS OUT display_manager,

--1 flight_control,

-1 panel_logic

--1 IN panel_logic.on

-1 OUT panel_logic =

-1 transition(IN panel_logic,

-1 att_cws_switch,*,*)

-1 OUT FORALL ss: Sensor_state: :

- - look (display_manager,ss) =

- 1 display (panel_logic,ss)

--1 OUT FORALL md:mode : :

--1 fc_engaged(md,flight_control) =

--1 engaged(md,panel_logic)

--1 END WHERE; 


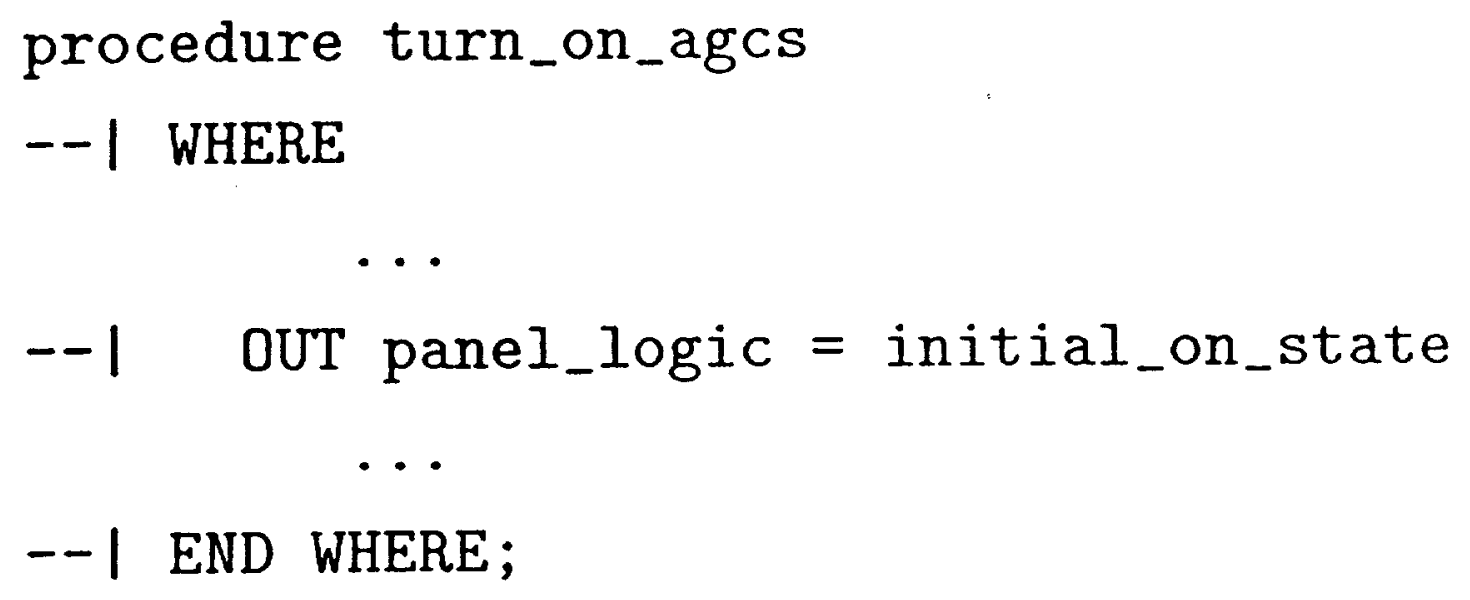

